\title{
Impact of the new european paediatric regulatory framework on ethics committees: overview and perspectives
}

\author{
A Altavilla', C Manfredi' , P Baiardi ${ }^{2}$, M Dehlinger-Kremer ${ }^{3}$, P Galletti ${ }^{4}$, A Alemany Pozuelo ${ }^{5}$, Chaplin ${ }^{6}$, A Ceci (aceci@ cvbf.net) ${ }^{2}$ \\ 1.Université de la Méditerranée - Espace Ethique Méditerranéen, Marseille, France \\ 2.Consorzio per Valutazioni Biologiche e Farmacologiche, Pavia, Italy \\ 3.Omnicare Clinical Research, Bad Soden a Ts., Germany, and Paediatric Working Group of EUCROF, the Netherlands \\ 4.Pierrel Research Italy Spa, Sesto San Giovanni, Italy \\ 5.TFS Trial Form Support, S.L., Madrid, Spain \\ 6.Göteborg Pediatric Growth Research Center, The Sahlgrenska Academy at the University of Gothenburg, Göteborg, Sweden
}

\section{Keywords \\ Ethics, Ethics committees, Paediatric clinical research, RESPECT, Survey, TEDDY}

\section{Correspondence}

A Ceci, Consorzio per Valutazioni Biologiche e Farmacologiche, Via Luigi Porta 14, 27100 Pavia, Italy. Tel: +39-0382-25075 |

Fax +39-0382-536544

Email: aceci@cvbf.net

\section{Received}

6 April 2011; revised 28 June 2011 ; accepted 1 July 2011

DOI:10.1111/j.1651-2227.201 1.02401.x

\begin{abstract}
Aim: To evaluate the impact of the new European paediatric regulatory framework on the activities of Ethics Committees operating in Europe and to assess their involvement and interest in paediatric research.

Methods: Task-force in Europe for Drug Development for the Young Network of Excellence and Relating Expectations and Needs to the Participation and Empowerment of Children in Clinical Trials project set up an inventory of Ethics Committees existing in Europe and conducted a survey on their approach to paediatric trials.

Results: Ethics Committees operating in 22 European Countries participated in this survey. Results showed a high lack of knowledge, understanding and awareness of the current European paediatric regulatory framework and a lack of involvement of Ethics Committees in paediatric research, especially in terms of training and education, demonstrated also by the decreasing number of Ethics Committees answering exhaustively to the whole questionnaire. The majority of participating Ethics Committees expressed interest in future initiatives related to paediatric research.

Conclusions: Despite a limited knowledge and understanding of the current paediatric regulatory framework, a significant number of Ethics Committees operating in Europe show interest in initiatives related to paediatric research. Networking may be an essential tool to be used to enhance Ethics Committees role in supporting paediatric research. Any initiative should be undertaken at European level in collaboration with European Union Institutions.
\end{abstract}

\section{INTRODUCTION}

Clinical research develops at an astonishing rate, and new drugs and therapeutic options are constantly discovered and applied in clinical practice.

Any type of scientific research on human subjects always has to take into account ethical guidelines and legal rules (1-3), and special attention and specific guarantees are required when 'vulnerable populations', such as children, are involved in clinical research (4-8).

In the European Union (EU; Europe), the rights and well-being of children participating in clinical research are currently assured by the provisions of Directive 2001/ 20/EC (CT-Dir) (9), a reference legislative instrument

\section{Key notes}

- A survey was carried out among EU ethics committees to evaluate the impact of the new European paediatric regulatory framework and to assess their involvement and interest in paediatric research. Results showed a lack of knowledge, understanding, awareness and involvement of ethics committees in paediatric research.

- Networking may be an essential tool to be used to enhance the role of ethics committees in supporting paediatric research. 
which entered into force in 2001 and aimed at providing a homogeneous legal and ethical framework for the conduct of clinical trials in the European Economic Area. The Directive includes a specific article, Article 4, aimed at ensuring the protection of minors taking into account their emotional, physiological and psychological specificities.

Between 2006 and 2007, the Task-force in Europe for Drug Development for the Young (TEDDY), a Network of Excellence funded by the European Commission under the 6th Framework Programme, carried out a survey to examine the measures enforced by Member States (MS) to implement the CT-Dir and other relevant European norms. Results showed that many differences exist in the protection of minors involved in clinical trials across Europe mainly because of the Directive implementation process and a lack of coordination among MS $(10,11)$.

In January 2007, the European Paediatric Regulation $(12,13)$ entered into force with the aim of increasing availability of medicines specifically studied in children, stimulating high-quality, ethical paediatric research, and making information and data on clinical trials in children and paediatric medicines easily accessible to the public (14). The Regulation is binding in its entirety and directly applicable in all Member States.

In February 2008, the European Commission released the 'European Ethical recommendations for clinical trials on medicinal products conducted with the paediatric population' (European Ethical Recommendations) aiming at developing safe and effective medicines for children while ensuring their protection and defining rules related to the risk/benefit balance assessment, the information and consent/assent process, and the process of ethical review of paediatric protocols (15). Individual data protection and insurance issues are also addressed. This nonbinding, declaratory instrument provides recommendations on ethical aspects of clinical trials involving children and introduces a new ethical and regulatory context integrating principles contained in various other European/international ethical/legal sources $(16,17)$. It constitutes a reference document for Ethics Committees (ECs).

Because the approval of clinical trials, including their ethical review, is performed at Member State level by national or local Ethics Committees, the correct implementation of the European regulatory framework (especially of the European Ethical Recommendations) is also under their responsibility and it directly influences their activities.

\section{AIM OF THE STUDY}

TEDDY and the Relating Expectations and needs to the Participation and Empowerment of children in Clinical Trials (RESPECT) project carried out a survey specifically addressed to ECs with the objective of pointing out proposals to facilitate the integration of Ethics Committees interested in paediatric research across Europe and to determine a road map to enhance the role of ECs in promoting the development of medicine tailored for children.
In particular, this investigation aimed at

- identifying Ethics Committees operating in Europe;

- identifying the ECs entitled to review paediatric protocols, according to their national legislation;

- evaluating how paediatric expertise is guaranteed in Ethics Committees;

- assessing ECs awareness of the new European paediatric regulatory framework;

- monitoring and assessing the impact of the new paediatric regulatory framework on Ethics Committees activities; and

- identifying future initiatives aimed at increasing ECs involvement in paediatric research.

\section{METHODOLOGY}

An inventory was carried out to identify Ethics Committees operating in Europe. The results of the first TEDDY survey $(10,11)$ provided the approximate number of ECs and the website addresses where their contact details are available. Other sources were TEDDY and RESPECT Partners, MS Medicines Agencies and personal direct information. When details were not available or easily accessible through the Internet, Paediatric Committee (PDCO) members were contacted for support. The inventory was planned and carried out between April and June 2009 and is continuously updated.

The survey was performed entirely online. ECs received an electronic invitation letter with a link to the questionnaire that presented first a filter question to establish whether the Committee was in charge of reviewing paediatric clinical trial protocols. ECs that are not entitled to assess paediatric studies did not continue with the investigation.

A total of 12 questions, whose replies were either mandatory or optional, were presented. The survey was divided into two main sections:

1. Ethics Committees and paediatric research under the new regulatory framework in Europe, focusing on the ECs awareness and knowledge of the current regulatory framework in paediatrics and its impact on their activities.

2. Interest and involvement of Ethics Committees in paediatric research, focusing on the interest of ECs in being involved in activities related to paediatric research.

The preliminary version of the questionnaire was discussed and shared with members of the PDCO. Two electronic mailings of the invitation were sent, and subsequently, Ethics Committees were contacted directly.

The results below are expressed as frequencies and percentages. Analyses were performed on the whole sample and on subgroups stratified in old EU Member States - EU15 (Austria, Belgium, Denmark, Finland, France, Germany, Ireland, Italy, Luxembourg, Portugal, Spain, Sweden, The Netherlands, UK) and new MS (Cyprus, Czech Republic, Estonia, Latvia, Malta, Poland, Slovakia). Norway and Iceland are associated with the European Research Programmes 


\begin{tabular}{|c|c|c|c|}
\hline Country & No. of ECs & $\begin{array}{l}\text { Inhabitants } \\
\text { (millions) }\end{array}$ & No. ECs/1.000.000 inh. \\
\hline Bulgaria & 103 & 7.6 & 13.55 \\
\hline Iceland & 3 & 0.3 & 10.00 \\
\hline Finland & 25 & 5.3 & 4.72 \\
\hline Italy & 270 & 60 & 4.50 \\
\hline Belgium & 38 & 10.7 & 3.55 \\
\hline Austria & 27 & 8.3 & 3.25 \\
\hline Spain & 143 & 45.8 & 3.12 \\
\hline Ireland & 13 & 4.5 & 2.89 \\
\hline Slovakia & 13 & 5.4 & 2.41 \\
\hline UK & 143 & 61.7 & 2.32 \\
\hline Latvia & 5 & 2.3 & 2.17 \\
\hline Luxembourg & 1 & 0.5 & 2.00 \\
\hline Malta & 1 & 0.5 & 2.00 \\
\hline The Netherlands & 32 & 16.4 & 1.95 \\
\hline Denmark & 9 & 5.5 & 1.64 \\
\hline Estonia & 2 & 1.3 & 1.54 \\
\hline Norway & 7 & 4.7 & 1.49 \\
\hline Poland & 54 & 38.1 & 1.42 \\
\hline Cyprus & 1 & 0.8 & 1.25 \\
\hline Czech Republic & 9 & 10.5 & 0.86 \\
\hline Sweden & 7 & 9.2 & 0.76 \\
\hline Germany & 54 & 82 & 0.66 \\
\hline France & 40 & 64.3 & 0.62 \\
\hline Lithuania & 2 & 3.3 & 0.61 \\
\hline Slovenia & 1 & 2 & 0.50 \\
\hline Hungary & 1 & 10 & 0.10 \\
\hline Portugal & 1 & 10.6 & 0.09 \\
\hline Greece & 1 & 11.2 & 0.09 \\
\hline Romania & 1 & 21.5 & 0.05 \\
\hline Total & 1007 & 504.3 & 2.00 \\
\hline
\end{tabular}

Sources: TEDDY inventory; European Union website (http://europa.eu/ about-eu/member-countries/index_en.htm)

and have been included in the EU-15 group because of their clinical research legal and ethical frameworks.

\section{RESULTS}

One thousand and seven ECs in 29 European Countries were identified (Table 1), and contact details were collected for more than 830 ECs in 28 Countries. Replies were gathered from a total of 154 ECs (18.2\%) operating in 22 Countries, with a response rate below $10 \%$ in four Countries (Spain, Finland, Germany, UK) but exceeding 30\% in 12 MS.

One hundred and thirty-nine Committees declared to be entitled of reviewing paediatric clinical protocols, 73 $(52.5 \%)$ of which also answered the optional questions.

Almost 59\% of the 73 responding ECs include paediatric experts as full members, usually a paediatrician, while $28.8 \%$ take advise from an external expert case by case (Fig. 1).

Only $14.4 \%$ of 139 ECs declared to having formally discussed and/or analysed the European Paediatric Regulation and only about $13 \%$ reported to having formally discussed and/or implemented the European Ethical

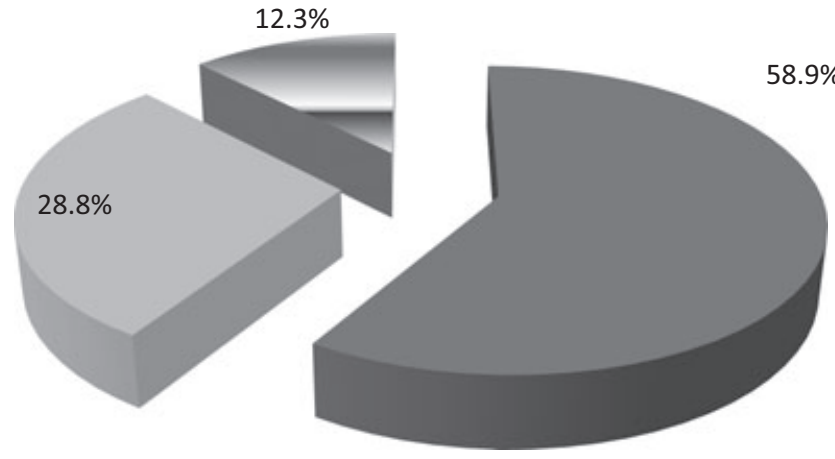

- Paediatric expertises included in the ethics committee

$\square$ Advice from external expert requested case by case

$=$ No paediatric expertise

Figure 1 Paediatric expertise in Ethics Committees.

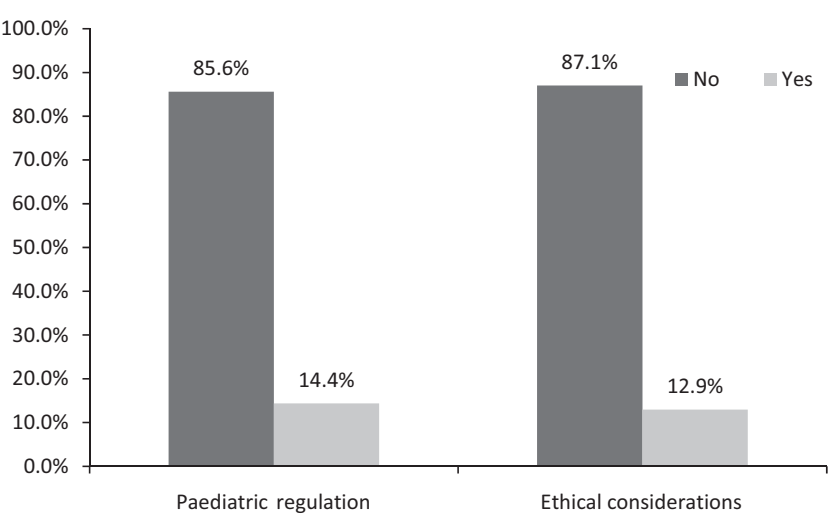

Figure 2 Ethics Committees formal knowledge of the current European paediatric framework.

Recommendations (Fig. 2). Analyses were usually carried out through dedicated sessions or training initiatives.

When asked about the influences of the new regulatory framework, 35.6\% of the 73 responding ECs reported 'no impact' on their work from the Paediatric Regulation, about $29 \%$ indicated 'low impact', 26\% 'sufficient' and around $10 \%$ 'high impact'. The trend was confirmed when considering the influence of the European Ethical Recommendations: only 6.8\% acknowledged 'high impact', 11.8\% 'sufficient', $26 \%$ and about $44 \%$ 'low' or 'none', respectively. Figure 3 details the major type of influences recognized by ECs. Other effects were increased attention to paediatric protocols, increased facility to carry out paediatric trials and the necessity to specify ethical requirements.

Figure 4 summarizes ECs opinions on the major issues to be dealt with under the new framework: about half of our sample identified the increased need for additional expertise to evaluate paediatric protocols $(52.1 \%)$ and for measures to minimize pain, distress and fear of children (46.6\%). 


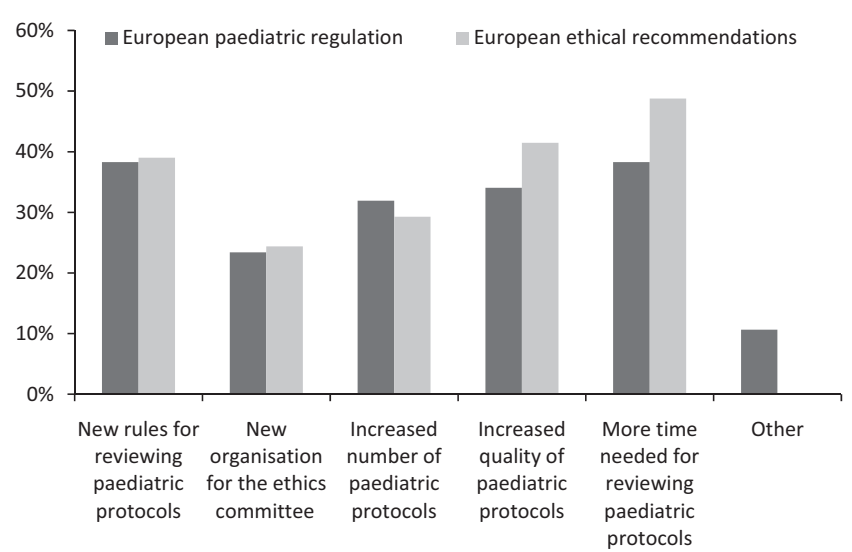

Figure 3 Impact of the European Paediatric Regulation and the European Ethical Recommendations.

Complexity in evaluating inclusion/exclusion criteria, risk/benefit balance and consent/assent procedures was also highlighted as main concerns. Issues related to compensation for parents and children and to insurance, provisions for protection of personal data and administrative burdens were declared to be unchanged.

Only 30\% per cent of the 73 respondents declared to have participated in initiatives in the field of paediatric research, and those who did usually took part in conferences $(68 \%)$ and training activities (32\%). Moreover, 74\% declared an interest in being involved in European initiatives related to paediatric research, preferring means such as training at national and local level and networking among ECs (59\% and 56\%, respectively). Debates and conferences (at national level) and educational initiatives supported by European Institutions were less preferred (54\% and 50\%, respectively). Seventy-three per cent of those Ethics Committees interested in networking belong to the EU-15 (Belgium, France, Germany, Ireland, Italy, Luxembourg, Portugal, Spain, Sweden, The Netherlands), while 27\% are established in new MS (Cyprus, Czech Republic, Estonia, Latvia, Malta, Poland).

\section{DISCUSSION}

The CT-Dir introduced a number of measures to harmonize the ethical review of clinical trials and facilitate clinical research. It required MS to legally establish Ethics Committees and introduced ECs obligations and specifications, formal procedures and timelines, composition and competencies. Specific provisions were also adopted for reviewing clinical trial protocols including children. However, because of the nature and legal force of a Directive, MS had some flexibility in implementing its provisions in their national legislation. Thus, ethical review procedures and the amount and quality of publicly available information vary significantly among European Countries (18).

Our results show that the number, competence and composition of ECs vary greatly across Europe. For example, Italy has the largest number of Ethics Committees, while Countries like Malta, Cyprus or Hungary have only one EC (Table 1). Nevertheless, if we relate the number of ECs to the population, Bulgaria has the highest number of ECs per million inhabitants (13.55) and Romania the lowest (0.05). These differences could reflect the diverse legal and social backgrounds and the different organization and funding of healthcare systems across the EU. An inventory of Ethics Committees operating in Europe, updated on a regular basis, is an important tool to facilitate the exchange of adequate and reliable information on their activities and procedures.

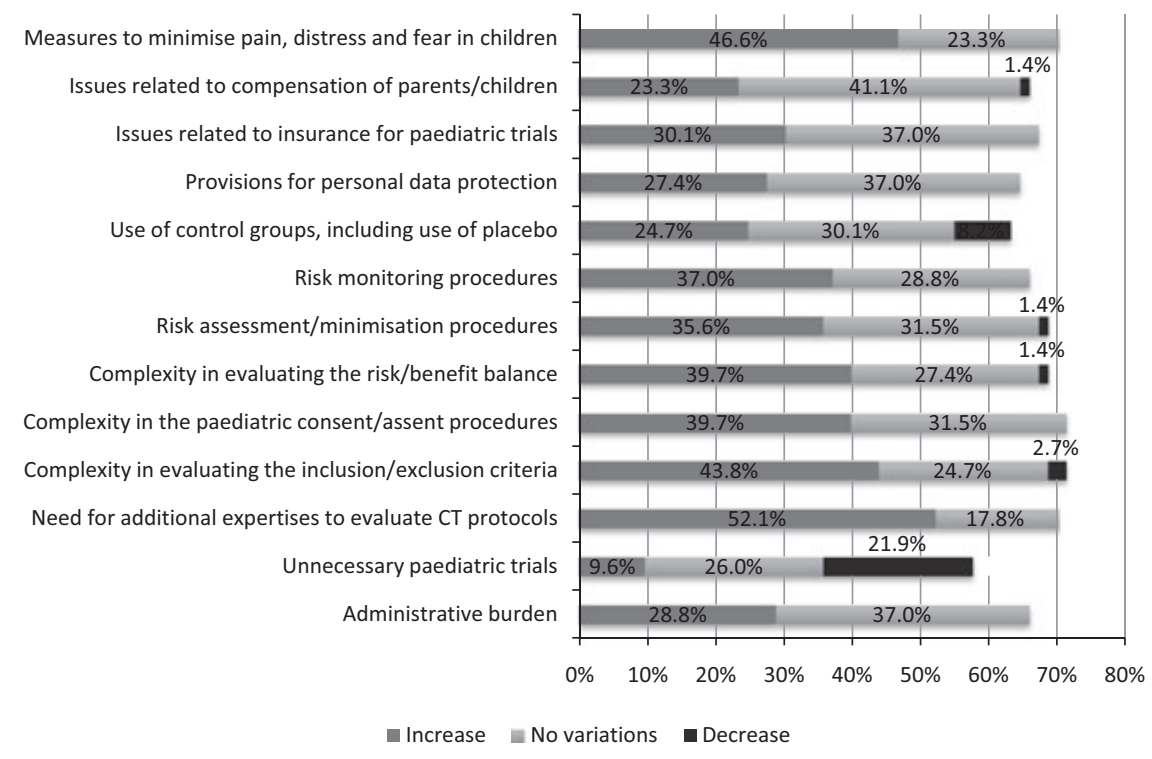

Figure 4 Main issues to be dealt with by Ethics Committees. 
Moreover, our results demonstrate that a gap exists between the current regulatory framework and ECs awareness, knowledge and understanding of the major issues related to paediatric clinical research: a very limited number of Ethics Committees declared of having formally discussed and analysed the most important European legal instruments devoted to paediatric research. That could explain the lower rate of ECs answering the optional questions related to more specific issues.

Additionally, the majority of ECs operating in EU-15 Member States declared a low impact of the Paediatric Regulation (39\%) and the European Ethical Recommendations (50\%). On the other hand, 33\% of ECs in new EU MS declared 'high impact' of the Paediatric Regulation and are mainly divided between 'high' and 'sufficient' impact of the European Ethical Recommendations, suggesting that the latter has been identified as a more effective tool for influencing the activities of Ethics Committees. These data suggest that ECs operating in new EU MS are more actively involved in efforts for integration and harmonization towards EU research and health norms and systems than EU-15 Ethics Committees (19).

Overall, ECs recognized as possible effects of the new European paediatric regulatory framework, the increase in the number of medicines tailored for children, of welldesigned paediatric trials and of paediatric multicentre clinical studies. Nevertheless, it has been stressed that there still is a lack of knowledge regarding the risks and burdens that are acceptable for children in different age groups.

Finally, even if the increased involvement of children in clinical research has been recognized as an effect of the new regulatory framework, it has also been underlined that it is difficult to adapt information to parents and children in accordance with the new requirements.

\section{CONCLUSIONS}

Our survey demonstrates that there is a lack of knowledge of the European paediatric regulatory framework, of awareness of ethical issues related to paediatric research and of involvement of Ethics Committees in paediatric research, especially in terms of training, education and other similar activities.

Given that ECs are one of the most important actors in guaranteeing the safety, rights and well-being of children involved in clinical research, it is of primary importance to increase their competence and their involvement in paediatric research and to promote the implementation of the European Ethical Recommendations at local level.

In this context, to increase the 'capacity building' in the field of ethics of paediatric clinical research should be an important objective.

Networking may be a fundamental tool to enhance collaboration and experiences and information exchange. It should be particularly important to promote these initiatives in the new Member States where the number of clinical trials is increasing.
One possible relevant result of networking could be the development of a comprehensive guide practically addressing paediatric ethical issues in accordance with all the relevant international and European ethical and legal sources.

This guide, chaired at EMA level, should address all those specific ethical issues related to paediatrics: information/authorization-assent process, paediatric expertise of Ethics Committees in charge of reviewing paediatric protocols (including training and education of the members of the ECs), use of placebo, compensation for damage as well as other specific aspects to be considered in reviewing paediatric protocols.

\section{ACKNOWLEDGEMENTS}

This study was carried out as a collaboration between TEDDY Network of Excellence supported by the EC Sixth Framework Programme (Contract n. 0005216 LSHBCT2005-005126) and RESPECT, a project supported by the EC Seventh Framework Programme (Contract n. 201938 COOP-HEALTH-2007-A). The Paediatric Working Group of EUCROF - the European CROs Federation, also cooperated. Authors thank Franco Bartoloni (IRIDIA Srl), Nicolas Thevenet (Euraxi Pharma, France), Jürgen Schaefer (Conreso GmbH, Germany), Anna Jurczynska (Quantum Experimental, Spain), Antoine Cournot (RPS, France), Philippa Smit-Marshall (the Netherlands) and the Spanish Association of Clinical Research Organizations for their invaluable support in the successful realization of this survey.

\section{References}

1. Huriet C. Introduction. In: Ethical Eye: Biomedical research, Council of Europe, 2004, 17.

2. European Medicines Agency. ICH Topic E 6 (R1) Guideline for Good Clinical Practice, Step 5 Note for Guidance on Good Clinical Practice. Doc. Ref. CPMP/ICH/135/95 2002 July. Available at URL http://www.ema.europa.eu/docs/en_GB/ document_library/Scientific_guideline/2009/09/ WC500002874.pdf (accessed 25 March 2011).

3. Commission of the European Communities. Commission Directive 2005/28/EC of 8 April 2005 laying down principles and detailed guidelines for good clinical practice as regards investigational medicinal products for human use, as well as the requirements for authorisation of the manufacturing or importation of such products. OJEU 2005; L91: 13-9.

4. European Commission Enterprise Directorate General. Better Medicines for Children. Proposed regulatory actions on paediatric medicinal products. Consultation document. Brussels, 2002.

5. Gill D, Crawley FP, LoGiudice M, Grosek S, Kurz R, de Lourdes-Levy M, et al. Guidelines for informed consent in biomedical research involving paediatric populations as research participants. Eur J Pediatr 2003; 162: 455-8.

6. John JE. The child's right to participate in research: myth or misconception? Br J Nurs 2007; 16: 157-60.

7. Knox CA, Burkhart PV. Issues related to children participating in clinical research. J Pediatr Nurs 2007; 22: 310-8.

8. International Conference on Harmonisation of Technical Requirements for Registration of Pharmaceuticals for Human Use. ICH Harmonised Tripartite Guideline - Clinical 
Investigation of Medicinal Products in the Pediatric Population- E11. Step 420 July 2000. Available at URL http://www.ich.org/fileadmin/Public_Web_Site/ICH_Products/Guidelines/ Efficacy/E11/Step4/E11_Guideline.pdf (accessed 15 June 2011).

9. European Parliament and the Council of the European Union. Directive 2001/20/EC of the European Parliament and of the Council of 4 April 2001 on the approximation of the laws, regulations and administrative provisions of the Member States relating to the implementation of good clinical practice in the conduct of clinical trials on medicinal products for human use. OJEC 2001; L121: 34-44.

10. Altavilla A, Giaquinto C, Ceci A. European survey on ethical and legal framework of clinical trials in paediatrics: results and perspectives. J Int Bioethique 2008; 19: 17-48.

11. Altavilla A, Giaquinto C, Giocanti D, Manfredi C, Aboulker JP, Bartoloni F, et al. Activity of ethics committees in Europe on issues related to clinical trials in paediatrics: Results of a survey. Pharmaceuticals Policy and Law 2009; 11: 79-87.

12. European Parliament and the Council of the European Union. Regulation (EC) No 1901/2006 of the European Parliament and of the Council of 12 December 2006 on medicinal products for paediatric use and amending Regulation (EEC) No 1768/92, Directive 2001/20/EC, Directive 2001/83/EC and Regulation (EC) No 726/2004. Official Journal of the European Union 2006; L378: 1-19.

13. European Parliament and the Council of the European Union. Regulation (EC) n. 1902/2006 of the European Parliament and of the Council of 20 December 2006 amending Regulation $1901 / 2006$ on medicinal products for paediatric use. Official Journal of the European Union 2006; L378: 20-1.
14. Saint-Raymond A, Seigneuret N. The European paediatric initiative: 1 year of experience. Paediatr Drugs 2009; 11: 9-10.

15. European Commission's Directorate-General for Health and Consumers. Ethical recommendations for clinical trials on medicinal products conducted with the paediatric population. Recommendations of the ad hoc group for the development of implementing guidelines for Directive 2001/20/EC relating to good clinical practice in the conduct of clinical trials on medicinal products for human use. Available at URL http://ec.europa.eu/health/documents/eudralex/vol-10/ (accessed 25 March 2011).

16. Council of Europe. Convention for the protection of Human Rights and dignity of the human being with regard to the application of biology and medicine: Convention on Human Rights and Biomedicine. CETS No.: 164. Available at: https:// www.sps-ops.sps.co.in/webdav/ja_ops.asp?rfp=fysceci (accessed 14 July 2011).

17. Council of Europe. Additional Protocol to the Convention on Human Rights and Biomedicine, concerning Biomedical Research. CETS No.: 195. Available at: http://conventions. coe.int/Treaty/Commun/QueVoulezVous.asp?NT=195\& $\mathrm{CL}=\mathrm{ENG}$ (accessed 14 July 2011).

18. European Forum for Good Clinical Practice (EFGCP) Ethics Working Party. The Procedure for the Ethical Review of Protocols for Clinical Research Projects in the European Union. Last update April 2010. Available at URL http://www.efgcp.be/EFGCPReports.asp?L1=5\&L2=1 (accessed 25 March 2011).

19. Glasa J. Establishment and work of ethics committees in central and eastern European countries. Med Etika Bioet 2002; 9: $9-12$. 